\title{
Deformations of real rational dynamics in tropical geometry
}

\author{
Tsuyoshi Kato
}

\section{Introduction}

Discrete dynamics defined by a rational function $f\left(z_{0}, \ldots, z_{n-1}\right)$ is a sequence $\left\{z_{N}\right\}_{N=0}^{\infty}$ satisfying the relation $z_{N}=f\left(z_{N-n}, \ldots, z_{N-1}\right)$ with initial data $\left(z_{0}, \ldots, z_{n-1}\right)$. In this paper we study dynamical properties of orbits $\left\{z_{N}\right\}_{N}$ given by rational functions by use of some comparison method in tropical geometry. From a dynamical view point, tropical geometry uses a kind of scale transform, and it provides with a connection between piecewise linear and rational functions with real coefficients.

A $(\max ,+)$-function $\varphi$ is a piecewise linear function of the form:

$$
\varphi(\bar{x})=\max \left(\alpha_{1}+\bar{j}_{1} \bar{x}, \ldots, \alpha_{m}+\bar{j}_{m} \bar{x}\right), \quad \bar{j}_{l} \bar{x}=\Sigma_{i=1}^{n} j_{l}^{i} x_{i}
$$

where $\bar{x}=\left(x_{1}, \ldots, x_{n}\right) \in \mathbb{R}^{n}, \bar{j}_{l}=\left(j_{l}^{1}, \ldots, j_{l}^{n}\right) \in \mathbb{Z}^{n}$ and $\alpha_{i} \in \mathbb{R}$. Correspondingly the parametrized rational function is given by:

$$
f_{t}(\bar{z})=\Sigma_{k=1}^{m} t^{\alpha_{k}} \bar{z}^{\bar{j}_{k}}, \quad \bar{z}^{\overline{j_{k}}}=\prod_{i=1}^{n} z_{i}^{j_{k}^{i}}
$$

where $\bar{z}=\left(z_{1}, \ldots, z_{n}\right) \in \mathbb{R}_{>0}^{n}=\left\{\left(w_{1}, \ldots, w_{n}\right): w_{i}>0\right\}$. These two functions admit one to one correspondence between their presentations. In fact the defining equations are transformed by taking conjugates by $\log _{t}$ and by letting $t \rightarrow \infty$. In some cases such $f_{t}$ are $t$ independent. We say that rational functions of the above form are elementary. For example $f(z)=2 z$ corresponds to $\max (x, x)$ and so is elementary in our sense, but $f(z)=\frac{1}{2} z$ is not the case. 
These two $\varphi$ and $f_{t}$ are connected passing through some intermediate functions $\varphi_{t}$, which Maslov inroduced as dequantization of the real line $\mathbb{R}([\mathrm{LM}],[\mathrm{Mi}])$. For $t>1$, there is a family of semirings $R_{t}$ which are all the real number $\mathbb{R}$ as sets. The multiplications and the additions are respectively given by $x \oplus_{t} y=\log _{t}\left(t^{x}+t^{y}\right)$ and $x \otimes_{t} y=x+y$. As $t \rightarrow \infty$ one obtains the equality:

$$
x \oplus_{\infty} y=\max (x, y) .
$$

Corresponding to the usual one on real numbers, one has $R_{t}$-polynomials $\varphi_{t}(\bar{x})=\oplus_{t}\left(\alpha_{k}+\bar{j}_{k} \bar{x}\right) . \varphi$ and $\varphi_{t}$ are connected as $\lim _{t \rightarrow \infty} \varphi_{t}=\varphi$, and it satisfies a $(\max ,+)$ equation $\varphi_{\infty}(\bar{x})=\max \left(\alpha_{1}+\bar{j}_{1} \bar{x}, \ldots, \alpha_{m}+\bar{j}_{m} \bar{x}\right)$.

Notice that all these $\varphi, \varphi_{t}$ and $f_{t}$ have one to one correspondences among their presentations. Let us relate $\varphi_{t}$ with $f_{t}$. Let $\log _{t}: \mathbb{R}_{>0}^{n} \rightarrow$ $\mathbb{R}^{n}$ be $\left(x_{1}, \ldots, x_{n}\right)=\left(\log _{t} z_{1}, \ldots, \log _{t} z_{n}\right)$.

Proposition $1.1(\mathrm{LM}, \mathrm{V}) \cdot f_{t} \equiv\left(\log _{t}\right)^{-1} \circ \varphi_{t} \circ \log _{t}: \mathbb{R}_{>0}^{n} \rightarrow(0, \infty)$ is a parametrized rational function $f_{t}(\bar{z})=\sum_{k=1}^{m} t^{\alpha_{k}} \bar{z}^{\bar{j}_{k}}$.

As $t \rightarrow \infty$, $\log _{t}$ becomes 'very contracting' maps, while $\varphi_{t}$ approaches to $\varphi$, but limit of $\log _{t}$ degenerates and just the constant. Such feature causes interesting phenomena from dynamical view points. In this paper, we study and compare two dynamics $\left\{x_{N}\right\}_{N}$ and $\left\{z_{N}\right\}_{N}$ given by:

$$
x_{N}=\varphi\left(x_{N-n}, \ldots, x_{N-1}\right), \quad z_{N}=f_{t}\left(z_{N-n}, \ldots, z_{N-1}\right)
$$

with the initial data $x_{0}=\log _{t} z_{0}, \ldots, x_{n-1}=\log _{t} z_{n-1}$. Notice that any orbits $z_{N}>0$ are given by positive real numbers for elementary $f_{t}$. In order to do this, we use the intermediate dynamics:

$$
x_{N}^{\prime}=\varphi_{t}\left(x_{N-n}^{\prime}, \ldots, x_{N-1}^{\prime}\right)
$$

parametrized by $t$ with the same initial data $x_{0}^{\prime}=\log _{t} z_{0}, \ldots, x_{n-1}^{\prime}=$ $\log _{t} z_{n-1}$. By the above proposition, two dynamics $\left\{z_{N}\right\}_{N}$ and $\left\{x_{N}^{\prime}\right\}_{N}$ are conjugate by $\log _{t}$. Since $\lim _{t \rightarrow \infty} \varphi_{t}=\varphi$ holds, one may expect that $\left\{z_{N}\right\}_{N}$ and $\left\{x_{N}\right\}_{N}$ may share some common dynamical properties.

An orbit $\left\{z_{N}\right\}_{N}$ given by $f_{t}$ is recursive, if there is some $M \geq 0$ so that $\left\{z_{N}\right\}_{N}$ is periodic with period $M . f_{t}$ is recursive if any orbits are the case for any initial values and any $t>1$. 
Proposition 1.2. Suppose an elementary rational function $f_{t}$ is recursive of period $M$. Then the corresponding $\varphi$ is also the same.

But it turns out that the converse is not true in general. Let us see by examples ([TI],[GKP],[HY]). Let us consider:

$$
\varphi(x, y)=\max (0, y)-x
$$

It is immediate to see that this is a recursive function of period 5 . Let us consider the corresponding function $f(z, w)=\frac{1+w}{z}$. Since $\varphi_{t}$ approaches to the recursive $\varphi$ as $t \rightarrow \infty$, one may expect that $f$ also gives a recursive function, which is true for this case. Now clearly the estimate $\varphi(x, y)=\max (0, y)-x \geq-x$ holds. So we have the equality as maps:

$$
\psi(x, y) \equiv \max (\varphi(x, y),-x)=\varphi(x, y)
$$

and in particular $\psi$ is also recursive. However the different presentation $\psi$ gives rise to another elementary rational function:

$$
g(z, w)=\frac{2+w}{z} .
$$

By easy calculations, it turns out that this is not recursive (for example try for the initial value $\left.\left(z_{0}, z_{1}\right)=(1,2)\right)$.

We have another example. Let us consider $\varphi(x, y)=\max (-y, y)-x$, which is a recursive function of period 9 . Let us consider the corresponding elementary rational function $f(z, w)=\frac{1+w^{2}}{z w}$. In this case also the estimate $\varphi(x, y)=\max (-y, y)-x \geq-x$ holds, and so we have the equality as $\operatorname{maps} \max (\varphi(x, y),-x)=\varphi(x, y)$. The corresponding function $g(z, w)=\frac{1+w+w^{2}}{z w}$ and $f$ above, both turn out not to be recursive (I thank to S.Tujimoto for computer calculations).

We would like to give a question that for a recursive $(\max ,+)$-function $\varphi$, whether one could find $\psi$ which is the same as $\varphi$ as maps, so that the corresponding elementary function $g_{t}$ is recursive. D. Takahashi told me that the answer is not known for the above example $f(z, w)=\frac{1+w^{2}}{z w}$.

These motivate to ask which dynamical properties are conserved with respect to two rational functions whose corresponding $(\max ,+)$ functions are the same as maps. 
Let $\varphi$ and $\psi$ be two ( $\max ,+)$-functions with $n$ variables. Then $\psi$ is equivalent to $\varphi$, if they are the same as maps, $\varphi\left(x_{1}, \ldots, x_{n}\right)=$ $\psi\left(x_{1}, \ldots, x_{n}\right)$ hold for all $\left(x_{1}, \ldots, x_{n}\right) \in \mathbb{R}^{n}$.

Definition 1.1. For two elementary functions $f_{t}$ and $g_{t}, g_{t}$ is a tropical deformation of $f_{t}$, if the corresponding $(\max ,+)$-functions $\varphi$ and $\psi$ are equivalent.

If the estimate $\varphi \geq \varphi^{\prime}$ holds, then $\psi=\max \left(\varphi, \varphi^{\prime}\right)$ and $\varphi$ are equivalent. For example $\frac{2+w}{z}$ is a tropical deformation of $\frac{1+w}{z}$. This example shows that in general tropical deformation does not imply topological conjugacy.

Tropically equivalent class $\left[f_{t}\right]$ is the set of elementary functions so that each element is a tropical deformation of $f_{t}$. In this paper we study dynamical properties of orbits, which are invariant under tropical deformations.

(A) Quasi recursive maps: Let $f_{t}$ be an elementary rational function and consider the dynamics $z_{N}=f_{t}\left(z_{N-n}, \ldots, z_{N-1}\right)$ with initial values $\left(z_{0}, \ldots, z_{N-1}\right) \in \mathbb{R}_{>0}^{n}$. If there are constants $C \geq 0$ and $M \geq 0$ independently of $t$ and initial values, so that the estimates:

$$
\max \left(\frac{z_{N+M}}{z_{N}}, \frac{z_{N}}{z_{N+M}}\right) \leq C, \quad N=0,1, \ldots
$$

hold, then we say that $f_{t}$ is a quasi recursive map of period $M$.

Our first result is:

Theorem 1.1. Suppose an elementary rational function $f_{t}$ corresponds to a $(\max ,+)$-function $\varphi$. Then $f_{t}$ is quasi recursive of minimum period $M$, if and only if $\varphi$ is recursive of the same minimum period.

The quasi recursive constant $C\left(f_{t}, M\right)$ is given by:

$$
C\left(f_{t}, M\right)=\sup _{t>1} \sup _{z_{0}, \ldots, z_{n-1}>0} \max \left(\frac{z_{N+M}}{z_{N}}, \frac{z_{N}}{z_{N+M}}\right) .
$$

We have additivity of quasi recursive maps. Let $f_{t}$ and $f_{t}^{\prime}$ be two elementary functions, and assume $f_{t}$ is quasi recursive. If the corresponding $(\max ,+)$-functions $\varphi$ and $\varphi^{\prime}$ satisfy the estimates $\varphi \geq \varphi^{\prime}$ for all points, then $f_{t}+f_{t}^{\prime}$ is also quasi recursive. 
Theorem 1.1 shows that a property to be quasi recursive is invariant under tropical deformations. More detailed analysis gives information on quasi recursive constants. Assume $\varphi$ and $\psi$ are equivalent and recursive. Let $f_{t}$ and $g_{t}$ be the corresponding quasi recursive functions respectively. If $\psi$ has the presentation as $\max \left(\alpha_{1}+\bar{j}_{1} \bar{x}, \ldots, \alpha_{m}+\bar{j}_{m} \bar{x}\right)$, then we say the number of the components of $g_{t}$ is $m=m_{g_{t}}$.

Corollary 1.1. There is a constant $C$ independent of choice of representatives $g_{t}$ in the tropical equivalent class $\left[f_{t}\right]$ so that we have a uniform bound on the quasi recursive constants of $g_{t}$ with respect to $m=m_{g_{t}}$ :

$$
C\left(g_{t}, M\right) \leq m^{C}
$$

When $\varphi$ is contracting (2.E) rather than recursive, correspondingly the rational dynamics by $f_{t}$ show some boundedness for their orbits:

Proposition 1.3. Suppose $\varphi$ is contracting. Then any orbits of $f_{t}$ are bounded away from both zero and infinity for all $t>1$.

Moreover there is some constant $C$ independent of $t$ and initial values, so that for any orbits $\left\{z_{N}\right\}_{N}$, there is some $N_{0}$ and for all $N \geq N_{0}$, the estimates hold:

$$
C^{-1} \leq z_{N} \leq C
$$

When $\varphi$ is contracting, then the above properties are invariant under tropical deformations, and so any tropical deformation $g_{t}$ of $f_{t}$ also satisfies the same properties. The constant $C$ above is also given as $m_{g_{t}}^{c}$ and $c$ is independent of choice of representatives $g_{t}$ in the tropical equivalent class $\left[f_{t}\right]$.

Proposition 1.3 is used to verify theorem 1.3 below.

Our comparison method works effectively when one of $z_{N}^{ \pm 1}$ are sufficiently large and the orbits contract as $\varphi$, while some fluctuation occurs if they are within some bounded regions.

(B) A characterization of tropical equivalence: We have an analytic characterization of tropical equivalence by the estimates on orbits. Let $f_{t}$ and $g_{t}$ be two elementary rational functions, choose any initial 
value $\left(z_{0}, \ldots, z_{n-1}\right)$, and denote the corresponding orbits by $\left\{z_{N}\right\}_{N}$ and $\left\{w_{N}\right\}_{N}$ for $f_{t}$ and $g_{t}$ respectively, where $w_{i}=z_{i}$ for $0 \leq i \leq n-1$. Let us put $m=\max \left(m_{f_{t}}, m_{g_{t}}\right)$.

Theorem 1.2. $f_{t}$ and $g_{t}$ are tropically equivalent, if and only if there exists a constant $C \geq 0$ independent of initial values and $t>1$ so that the double exponential estimates:

$$
\max \left(\frac{z_{N}}{w_{N}}, \frac{w_{N}}{z_{N}}\right) \leq m^{C^{N}}
$$

hold for all $N=0,1, \ldots$

(C) Perturbations of real rational dynamics: Let $\varphi\left(x_{0}, \ldots, x_{n-1}\right)$ be a $(\max ,+)$ function and put $\bar{x}_{0}=\left(x_{0}, \ldots, x_{n-1}\right)$. We regard it as a $\operatorname{map} \varphi: \mathbb{R}^{n} \rightarrow \mathbb{R}^{n}$ by $\varphi\left(\bar{x}_{0}\right)=\left(x_{1}, \ldots, x_{n-1}, \varphi\left(\bar{x}_{0}\right)\right)$. Let $L_{0}$ be a segment connecting $\bar{x}_{0}$ and $\varphi\left(\bar{x}_{0}\right)$ in $\mathbb{R}^{n}$, and put the connected piecewise linear line:

$$
L=\cup_{k=0}^{\infty} \varphi^{k}\left(L_{0}\right)
$$

We call it as a trace of $\varphi$. Structure of the traces contain some informations on the iterations by $\varphi$. For example if $\varphi$ is recursive, then any traces consist of closed piecewise linear lines ([TI]). Suppose $\varphi\left(x_{0}, x_{1}\right)$ has two variables, and is homogeneous so that 0 is a fixed point. Then degree of $\varphi$ around the origin is given by use of traces and the corresponding return maps.

In this paper we study small perturbations of real rational dynamics in two variables passing through tropical transforms into $(\max ,+)$ functions. Let $f_{t}\left(z_{0}, z_{1}\right)$ be an elementary rational function by two variables. For $i=0,1$, an $\epsilon_{i}$-perturbation is given by the following equation:

$$
z_{N+1}=z_{N-1+i}^{\epsilon_{i}} f_{t}\left(z_{N-1}, z_{N}\right) \equiv f_{t}^{\epsilon_{i}}\left(z_{N-1}, z_{N}\right), \quad \epsilon_{i} \in \mathbb{R} .
$$

Definition 1.2. A dynamics given by $f_{t}$ is stable, if any orbits are bounded away from both zero and infinity. It is unstable if there is a constant $C$ independent of $t$ so that for any initial value $\bar{z}_{0}$ with $\left|\bar{z}_{0}\right| \geq C$, the orbits are unbounded.

An $\epsilon_{i}$-perturbation is stationary, if it is stable for $\epsilon_{i}>0$ and unstable for $\epsilon_{i}<0$ for all sufficiently small $\left|\epsilon_{i}\right|<<1$. 
Let us consider a recursive map $z_{N+1}=f\left(z_{N-1}, z_{N}\right)=\frac{1+z_{N}}{z_{N-1}}$ whose orbits all have period 5 . In this paper we show the following:

Theorem 1.3. Let $g_{t}$ be any tropical deformation of $f(z, w)=\frac{1+w}{z}$. Then $\epsilon_{0}$ perturbations $g_{t}^{\epsilon}(z, w)=z^{\epsilon} g_{t}(z, w)$ are all stationary.

For example, $z_{N+1}=z_{N-1}^{\epsilon}\left(\frac{1+z_{N}}{z_{N-1}}+l \frac{1}{z_{N-1}}\right), l=0,1, \ldots$ are all stationary. Let $\varphi$ correspond to $f_{t}$ and put $\varphi^{\epsilon_{0}}\left(x_{0}, x_{1}\right)=\varphi\left(x_{0}, x_{1}\right)+\epsilon_{0} x_{0}$. Then $\varphi^{\epsilon_{0}}$ corresponds to $f_{t}^{\epsilon_{0}}$. The conclusion follows for any $f_{t}^{\epsilon_{0}}$ if the corresponding $\varphi^{\epsilon_{0}}$ satisfies properties that it is contracting for $\epsilon_{0}>0$ and is expanding for $\epsilon_{0}<0$. Such properties are obtained by chasing the traces directly for these perturbed $(\max ,+)$-functions. Such dynamical properties are independent of presentations of the $(\max ,+)$-functions.

It has a merit to analyze dynamical properties by replacing rational dynamics by piecewise linear one, since one has to know only finite set of points to recover $\varphi^{k}\left(L_{0}\right)$ above, since they are broken lines.

For $\epsilon_{0}>0$, any orbits for these dynamics are all bounded. On the other hand the method gives no information about such things when $\epsilon=0$. At present I do not know which dynamics of the form $z_{N+1}=$ $\frac{1+z_{N}}{z_{N-1}}+l \frac{1}{z_{N-1}}$ have the property that all orbits are bounded.

So far we have assumed that $(\max ,+)$ functions are of the form $\max \left(\alpha_{1}+\right.$ $\left.\bar{j}_{1} \bar{x}, \ldots, \alpha_{m}+\bar{j}_{m} \bar{x}\right)$, which is enough for our purpose. But here we do not use concavity of $(\max ,+)$ functions, and most of the results in this paper hold for functions of the form $\varphi(\bar{x})=\max \left(\alpha_{1}+\bar{j}_{1} \bar{x}, \ldots, \alpha_{m}+\right.$ $\left.\bar{j}_{m} \bar{x}\right)-\max \left(\beta_{1}+\bar{i}_{1} \bar{x}, \ldots, \beta_{l}+\bar{i}_{l} \bar{x}\right)$.

\section{Tropical transform}

2.A Estimates for orbits: Let $\varphi_{t}(x)=\oplus_{t}\left(\alpha_{k}+\bar{j}_{k} \bar{x}\right), \bar{x} \in \mathbb{R}^{n}, \bar{j}_{k} \in \mathbb{Z}^{n}$ be an $R_{t}$ polynomial, whose limit $t \rightarrow \infty$ gives a (max, + )-function $\varphi(\bar{x}) \equiv \varphi_{\infty}(\bar{x})=\max \left(\alpha_{1}+\bar{j}_{1} \bar{x}, \ldots, \alpha_{m}+\bar{j}_{m} \bar{x}\right)$. We say that $m$ is the number of the components of $\varphi$. It plays an important role for several estimates. 
Let us denote:

$$
a(t, \varphi)=\sup _{\bar{x} \in \mathbb{R}^{n}}\left|\varphi_{t}(\bar{x})-\varphi(\bar{x})\right| .
$$

Lemma 2.1. (1) $a(t, \varphi) \leq \log _{t} m \rightarrow 0, t \rightarrow \infty$.

(2) $\varphi$ is Lipschitz.

Proof: (1) We show the estimates $\left|x_{1} \oplus_{t} \cdots \oplus_{t} x_{m}-\max \left(x_{1}, \ldots, x_{m}\right)\right| \leq$ $\log _{t} m$. Assume $x_{1}=\max \left(x_{1}, \ldots, x_{m}\right)$. Then $x_{1} \oplus_{t} \cdots \oplus_{t} x_{m}=\log _{t}\left(t^{x_{1}}+\right.$ $\left.\cdots+t^{x_{m}}\right)=\log _{t}\left(t^{x_{1}}\left(1+t^{x_{2}-x_{1}}+\cdots+t^{x_{m}-x_{1}}\right)\right)=x_{1}+\log _{t}\left(1+t^{x_{2}-x_{1}}+\right.$ $\left.\cdots+t^{x_{m}-x_{1}}\right)$. Since $x_{i}-x_{1} \leq 0$ are non positive, the estimates $\log _{t}(1+$ $\left.t^{x_{2}-x_{1}}+\cdots+t^{x_{m}-x_{1}}\right) \leq \log _{t} m \rightarrow 0$ hold.

(2) Since $\varphi$ is piecewise linear, the conclusion is clear.

This completes the proof.

Let us consider the orbits given by $x_{N}=\varphi\left(x_{N-n}, \ldots, x_{N-1}\right)$ and $x_{N}^{\prime}=$ $\varphi_{t}\left(x_{N-n}^{\prime}, \ldots x_{N-1}^{\prime}\right)$ with the same initial value $x_{0}=x_{0}^{\prime}, \ldots, x_{n-1}=x_{n-1}^{\prime}$.

Lemma 2.2. Let $\left\{x_{N}\right\}_{N}$ and $\left\{x_{N}^{\prime}\right\}_{N}$ be as above, and $c$ be the Lipschitz constant of $\varphi$. There are some universal polynomials $P_{N}$ of degree $N-n$ so that the estimates hold:

$$
\left|x_{N}-x_{N}^{\prime}\right| \leq P_{N}(c) \log _{t} m
$$

where $m$ is the number of the components of $\varphi$. In particular for any small $\epsilon>0$ and large $N>>0$, there is a large $t_{0}>>0$ so that for all $t \geq t_{0}$, the estimates $\left|x_{k}-x_{k}^{\prime}\right|<\epsilon$ hold for all $0 \leq k \leq N$.

Proof: Let us denote $\bar{x}_{N}=\left(x_{N}, \ldots, x_{N+n-1}\right) \in \mathbb{R}^{n}$. Thus $x_{N+n}=$ $\varphi\left(\bar{x}_{N}\right)$ hold for all $N \geq 0$. Similar for $\bar{x}_{N}^{\prime}$.

Firstly one has the estimates $\left|x_{n}^{\prime}-x_{n}\right| \leq \log _{t} m$ by lemma 2.1(1).

Now since $\varphi$ is $c$ Lipschitz and $\bar{x}_{1}-\bar{x}_{1}^{\prime}=\left(0, \ldots, 0, x_{n}-x_{n}^{\prime}\right)$ holds, the estimates hold:

$$
\begin{aligned}
\left|x_{n+1}-x_{n+1}^{\prime}\right| & =\left|\varphi\left(\bar{x}_{1}\right)-\varphi_{t}\left(\bar{x}_{1}^{\prime}\right)\right| \\
& \leq\left|\varphi\left(\bar{x}_{1}\right)-\varphi\left(\bar{x}_{1}^{\prime}\right)\right|+\left|\varphi_{t}\left(\bar{x}_{1}^{\prime}\right)-\varphi\left(\bar{x}_{1}^{\prime}\right)\right| \\
& \leq c\left|\bar{x}_{1}-\bar{x}_{1}^{\prime}\right|+\log _{t} m \leq(c+1) \log _{t} m
\end{aligned}
$$


Next we have estimates:

$$
\begin{aligned}
\left|\varphi\left(\bar{x}_{2}\right)-\varphi\left(\bar{x}_{2}^{\prime}\right)\right| & \leq c\left(\left|x_{n+1}-x_{n+1}^{\prime}\right|+\left|x_{n}-x_{n}^{\prime}\right|\right) \\
& \leq c\{(c+1)+1\} \log _{t} m=c(c+2) \log _{t} m .
\end{aligned}
$$

Thus we have the following:

$$
\begin{aligned}
& \left|x_{n+2}-x_{n+2}^{\prime}\right|=\left|\varphi\left(\bar{x}_{2}\right)-\varphi_{t}\left(\bar{x}_{2}^{\prime}\right)\right| \\
& \quad \leq\left|\varphi\left(\bar{x}_{2}\right)-\varphi\left(\bar{x}_{2}^{\prime}\right)\right|+\left|\varphi\left(\bar{x}_{2}^{\prime}\right)-\varphi_{t}\left(\bar{x}_{2}^{\prime}\right)\right| \leq[c(c+2)+1] \log _{t} m
\end{aligned}
$$

The rest process is similar, and by iterating the same estimates, one finds some polynomials $P_{N}$ of degree $N-n$ so that the estimates $\mid x_{N}-$ $x_{N}^{\prime} \mid \leq P_{N}(c) \log _{t} m$ hold. This completes the proof.

Remark: One can choose a larger $c>1$ so that the estimates $P_{N}(c) \leq$ $((n+1) c)^{N-n}$ hold for all $N \geq n$.

2.B Characterization of tropical equivalences by orbits: Let $\varphi$ be a $(\max ,+)$-function and $f_{t}\left(z_{0}, \ldots, z_{n-1}\right)$ be the corresponding parametrized rational function. For intial values $\bar{z}_{0}=\left(z_{0}, \ldots, z_{n-1}\right)$, $z_{i}>0$, let $\left\{z_{N}\right\}_{N}$ be the orbits, and denote $\bar{z}_{N}=\left(z_{N}, \ldots, z_{N+n-1}\right) \in$ $\mathbb{R}_{>0}^{n}$. Thus $z_{N+n}=f_{t}\left(\bar{z}_{N}\right)$ hold for all $N \geq 0$.

Let $\varphi_{t}$ be the $R_{t}$ polynomials corresponding to $\varphi$, and consider the orbits $x_{N+n}^{\prime}=\varphi_{t}\left(\bar{x}_{N}^{\prime}\right)$, where $x_{i}^{\prime}=\log _{t}\left(z_{i}\right)$ for $0 \leq i \leq n-1$.

By proposition 1.1, the equalities:

$$
\log _{t}\left(z_{N+n}\right)=\log _{t}\left(f_{t}\left(\bar{z}_{N}\right)\right)=\varphi_{t}\left(\log _{t}\left(\bar{z}_{N}\right)\right)
$$

hold for all $N \geq 0$. In particular we have the equality:

$$
x_{N}^{\prime}=\log _{t}\left(z_{N}\right) \in \mathbb{R}, \quad N \geq 0 .
$$

Let $f_{t}$ and $g_{t}$ be two elementary rational functions. Let us choose an initial value $\left(z_{0}, \ldots, z_{n-1}\right)$, and denote the corresponding orbits $\left\{z_{N}\right\}_{N}$ and $\left\{w_{N}\right\}_{N}$ for $f_{t}$ and $g_{t}$ respectively, where we put the same initial value $w_{i}=z_{i}$ for $0 \leq i \leq n-1$. Let us put $m=\max \left(m_{f_{t}}, m_{g_{t}}\right)$, where $m_{f_{t}}$ is the number of the components of $f_{t}$. 
Theorem 2.1. $f_{t}$ and $g_{t}$ are tropically equivalent, if and only if there exists a constant $C \geq 0$ independent of initial values and $t>1$ so that the double exponential estimates:

$$
\max \left(\frac{z_{N}}{w_{N}}, \frac{w_{N}}{z_{N}}\right) \leq m^{C^{N}}
$$

hold for all $N=0,1, \ldots$

Proof: Let us take any initial value $z_{0}=w_{0}, \ldots, z_{n-1}=w_{n-1}$. Let $\varphi$ and $\psi$ be the $(\max ,+)$-functions corresponding to $f_{t}$ and $g_{t}$ respectively. For the initial value $x_{i}=y_{i}=\log _{t} z_{i}, 0 \leq i \leq n-1$, let us denote the corresponding orbits by $\left\{x_{N}\right\}_{N}$ and $\left\{y_{N}\right\}_{N}$. We also put $x_{N}^{\prime}=\log _{t}\left(z_{N}\right)$ and $y_{N}^{\prime}=\log _{t}\left(w_{N}\right)$ respectively. Thus $\left\{x_{N}^{\prime}\right\}_{N}$ is the orbit for $\varphi_{t}$ and $\left\{y_{N}^{\prime}\right\}_{N}$ is for $\psi_{t}$.

Now suppose $f_{t}$ and $g_{t}$ are tropically equivalent, and so $\varphi$ and $\psi$ are the same as maps. Then by lemma 2.2, the estimates:

$$
\left|x_{N}-x_{N}^{\prime}\right|,\left|y_{N}-y_{N}^{\prime}\right| \leq P_{N}(c) \log _{t} m
$$

hold. By the assumption, $x_{N}=y_{N}$ hold, and so we have the estimates:

$$
\log _{t}\left(\frac{z_{N}}{w_{N}}\right)^{ \pm} \equiv\left|\log _{t}\left(z_{N}\right)-\log _{t}\left(w_{N}\right)\right|=\left|x_{N}^{\prime}-y_{N}^{\prime}\right| \leq 2 P_{N}(c) \log _{t} m .
$$

One may assume $2 P_{N}(c) \leq C^{N}$ for some $C \geq 0$ by the remark at the end of 2.A. In particular we have the estimates:

$$
\left(\frac{z_{N}}{w_{N}}\right)^{ \pm} \equiv \max \left(\frac{z_{N}}{w_{N}}, \frac{w_{N}}{z_{N}}\right) \leq m^{C^{N}} .
$$

Let us verify the converse. Let us choose any initial value $x_{i}=y_{i}$ for $0 \leq i \leq n-1$. Then for large $t>>0$ we put the initial value by $z_{i}=w_{i}=t^{x_{i}}$. The estimates $\left(\frac{z_{N}}{w_{N}}\right)^{ \pm} \leq m^{C^{N}}$ imply the ones $\left|x_{N}^{\prime}-y_{N}^{\prime}\right| \leq$ $C^{N} \log _{t} m$ for all $N=0,1, \ldots$ By lemma 2.2, one has the estimates:

$$
\left|x_{N}-y_{N}\right| \leq 2 P_{N}(c) \log _{t} m+C^{N} \log _{t} m .
$$

Since $t$ are arbitrarily and the left hand side of the above estimate is independent of $t$, it follows by letting $t \rightarrow \infty$ that the equalities $x_{N}=y_{N}$ must hold. This completes the proof. 
2.C Comparison between orbits: Now we consider what happens for $\varphi$ when $f_{t}$ are recursive of period $M, z_{N+M}=z_{N}$ for all $N \geq 0$ and $t>1$. In this case we show that $\varphi$ also satisfies the same property. Later we will see that the converse is not the case, but still some 'quasi recursiveness' is satisfied. Notice that $f_{t}$ is recursive if for any initial values, the equalities $z_{j+M}=z_{j}$ hold for all $0 \leq j \leq n-1$.

Proposition 2.1. Let $\varphi$ be a (max, + )-function with the corresponding rational function $f_{t}$. Assume $f_{t}$ is recursive of period $M$. Then $\varphi$ is also the same.

Proof: Let $\left\{x_{N}\right\}_{N}$ and $\left\{x_{N}^{\prime}\right\}_{N}$ be the orbits of $\varphi$ and $\varphi_{t}$ with the same initial value $x_{i}$ for $0 \leq i \leq n-1$, and put $z_{i}=t^{x_{i}}$. For any small $\epsilon>0$, one can choose large $t>>1$ so that the estimates $\left|x_{i}-x_{i}^{\prime}\right| \leq \epsilon$ hold for $0 \leq i \leq n-1+M$ by lemma 2.2 .

By the assumption, $z_{j+M}=z_{j}$ hold for $0 \leq j \leq n-1$, and so $x_{j+M}^{\prime}=x_{j}^{\prime}$ also hold. Then we have the estimates:

$$
\left|x_{j+M}-x_{j}\right| \leq\left|x_{j+M}-x_{j+M}^{\prime}\right|+\left|x_{j+M}^{\prime}-x_{j}^{\prime}\right|+\left|x_{j}^{\prime}-x_{j}\right| \leq 2 \epsilon .
$$

Since the left hand side is independent of $t$ and $\epsilon>0$ is arbitrarily small, this shows that the equalities $x_{j+M}=x_{j}$ must hold. Thus $\varphi$ gives a recursive map of period $M$. This completes the proof.

Example: Let $f_{t}(z, w)=t \frac{w}{z}$. Then easy calculations show that it is recursive of period 6 . Then the corresponding ( $\max ,+$ )-function is $\varphi(x, y)=1+y-x$ which is also recursive of the same period.

2.D Uniform rates of orbits: Let $\varphi$ be a $(\max ,+)$-function and $f_{t}$ be the corresponding rational function. In general the converse of proposition 2.1 is not true, but we have the following:

Proposition 2.2. Suppose $\varphi$ is recursive of period $M$. Then there is a constant $C>0$ independent of $t$ so that for any initial value $z_{0}, \ldots, z_{n-1}>0$ and the orbit $\left\{z_{N}\right\}_{N}$ for $f_{t}$, the uniform estimates:

$$
\left(\frac{z_{N+M}}{z_{N}}\right)^{ \pm} \equiv \max \left(\frac{z_{N+M}}{z_{N}}, \frac{z_{N}}{z_{N+M}}\right) \leq C
$$

hold for all $N \geq 0$. 
Proof: It is enough to verify the conclusion for $0 \leq N \leq n-1$. Let $m$ be the number of the components of $\varphi$, and $\left\{x_{N}\right\}_{N}$ and $\left\{x_{N}^{\prime}\right\}_{N}$ be orbits for $\varphi$ and $\varphi_{t}$ respectively, where we put the intial value $x_{i}=x_{i}^{\prime}=\log _{t} z_{i}$ for $0 \leq i \leq n-1$. Then by lemma $2.2,\left|x_{N}-x_{N}^{\prime}\right| \leq P_{N}(c) \log _{t} m$ hold. Since $\varphi$ is recursive $x_{N+M}=x_{N}$, thus the estimates hold:

$$
\left(\frac{z_{N}}{z_{N+M}}\right)^{ \pm}=\left(\frac{t^{x_{N}^{\prime}}}{t^{x_{N+M}^{\prime}}}\right)^{ \pm}=t^{\left|x_{N}^{\prime}-x_{N+M}^{\prime}\right|} \leq t^{2 P_{N+M}(c) \log _{t} m}=m^{2 P_{N+M}(c)}
$$

Thus one can put $C=m^{2 P_{n-1+M}(c)}$.

This completes the proof.

Now we define the invariant of quasi recursive dynamics. Let $f_{t}$ be a parametrized rational function and $\varphi$ be the recursive map of period $M$. For initial values $z_{0}, \ldots, z_{n-1}$, let us denote the orbits of $f_{t}$ by $\left\{z_{N}\right\}_{N}$.

Definition 2.1. If there is a constant $C \geq 0$ and a number $M \geq 0$ which are both independent of $t$ and initial values so that the estimates $\left(\frac{z_{N+M}}{z_{N}}\right)^{ \pm} \leq C$ hold for all $0 \leq N$, then we say that $f_{t}$ is a quasi recursive map of period $M$. The quasi recursive constant is given by:

$$
C\left(f_{t}, M\right)=\sup _{t>1} \sup _{z_{0}, \ldots, z_{n-1}>0}\left(\frac{z_{N+M}}{z_{N}}\right)^{ \pm} .
$$

The constant $C$ in proposition 2.2 depends only on $\varphi$ as a map and the number of the components $m$ of $f_{t}$. The above proof gives a bound:

$$
C\left(f_{t}, M\right) \leq m^{2 P_{n-1+M}(c)} \text {. }
$$

Corollary 2.1. Let $\varphi$ be a recursive $(\max ,+)$-function of period $M$, and $f_{t}$ be the corresponding rational function. Then there is a constant $C>0$ so that for any tropical deformation $g_{t}$ of $f_{t}$ and any orbits $\left\{w_{N}\right\}_{N}$ for $g_{t}$, we have the uniform estimates:

$$
\max \left(\frac{w_{N}}{w_{N+M}}, \frac{w_{N+M}}{w_{N}}\right) \leq m^{C}
$$

for all $0 \leq N$, where $m$ is the number of the components of $g_{t}$.

Thus among the tropical equivalent class of $\varphi$, the minimum $m_{[\varphi]}$ of the numbers of the components determines the optimal estimate above.

Let us verify additivity of quasi recursive maps. 
Lemma 2.3. Let $f_{t}$ and $f_{t}^{\prime}$ be two elementary functions. Assume that the corresponding $(\max ,+)$-functions $\varphi$ and $\varphi^{\prime}$ satisfy the estimates $\varphi \geq \varphi^{\prime}$ for all points, and $\varphi$ is recursive.

Then $f_{t}+f_{t}^{\prime}$ is quasi recursive.

Proof: By the assumption, $f_{t}+f_{t}^{\prime}$ has the corresponding $(\max ,+)-$ function $\max \left(\varphi, \varphi^{\prime}\right)$. On the other hand, by the inequality $\varphi \geq \varphi^{\prime}$, $\max \left(\varphi, \varphi^{\prime}\right)=\varphi$ holds. In particular the former is also recursive. Thus the conclusion follows by corolalry 2.1 . This completes the proof.

Example: Let $f(z, w)=\frac{1+w^{2}}{z w}$. The corresponding $(\max ,+)$ function is given by $\varphi(x, y)=\max (-y, y)-x$, which is recursive of period 9 . Let $f^{\prime}(z, w)=\frac{1}{z}$, and $\varphi^{\prime}(x, y)=-x$. Then clearly the pointwise estimate $\varphi \geq \varphi^{\prime}$ holds, and so by lemma $2.3,\left(f+f^{\prime}\right)(z, w)=\frac{1+w+w^{2}}{z w}$ is quasi recursive. By computer calculation, it can be seen that both $f$ and $f+f^{\prime}$ are not recursive. By the same argument, $\left(f+l f^{\prime}\right)(z, w)=\frac{1+l w+w^{2}}{z w}$ are all quasi recursive for $l=0,1, \ldots$ They have the number of the components $m=l+2$.

Let us charecterize quasi recursive maps with respect to the corresponding recursive $(\max ,+)$-functions:

Theorem 2.2. Suppose an elementary $f_{t}$ corresponds to a $(\max ,+)$ function $\varphi$. Then $f_{t}$ is quasi recursive, if and only if $\varphi$ is recursive with the same minimum period.

Proof: If $\varphi$ is recursive, then $f_{t}$ is quasi recursive by proposition 2.2.

Let us verify the converse, and assume $f_{t}$ is quasi recursive of period $M$. Let us choose any initial value $\left(x_{0}, \ldots, x_{n-1}\right) \in \mathbb{R}^{n}$, and consider the orbit $\left\{x_{N}\right\}_{N}$ for $\varphi$. For large $t>>0$, let us put the initial value $z_{0}=t^{x_{0}}, \ldots, z_{n-1}=t^{x_{n-1}}$ and consider the orbit $\left\{z_{N}\right\}_{N}$ of $f_{t}$. By the assumption, there is a constant $C \geq 1$ independent of $z_{0}, \ldots, z_{n-1}$ so that the estimates $\left(\frac{z_{N+M}}{z_{N}}\right)^{ \pm} \leq C$ hold for all $N \geq 0$. Now put $x_{N}^{\prime}=\log _{t} z_{N}$. Then the estimates:

$$
\left|x_{N+M}^{\prime}-x_{N}^{\prime}\right| \leq \log _{t} C
$$


hold. By lemma 2.2, the estimates:

$$
\left|x_{N}-x_{N}^{\prime}\right| \leq P_{n-1+M}(c) \log _{t} m, \quad 0 \leq N \leq n-1+M
$$

hold for some constant $c$, where $m$ is the number of the components of $\varphi$. These two estimates imply that for any small $\epsilon>0$, there is a large $t_{0}$ so that for all $t \geq t_{0}$, the estimates

$$
\left|x_{N+M}-x_{N}\right|<\epsilon, \quad 0 \leq N \leq n-1
$$

hold. Since $\epsilon$ is arbitrary, this implies the equalities $x_{0}=x_{M}, \ldots, x_{n-1}=$ $x_{n-1+M}$ hold.

Finally we show that they have the same minimum period $M$. Suppose the minimum period of $f_{t}$ is $M$, and assume $\varphi$ has period $M^{\prime}<M$. Then the above proof shows that $f_{t}$ also must have the period $M^{\prime}$, which cannot happen. The converse also holds by the same argument. This completes the proof.

Corollary 2.2. Suppose $\varphi$ is recursive of the minimum period $M$. Then for the corresponding $f_{t}$ and any $1 \leq M^{\prime} \leq M-1$, the quasi recursive constants $C\left(f_{t}, M^{\prime}\right)$ are all infinity.

Proof: Otherwise, theorem 2.2 shows that $\varphi$ has period $M^{\prime}<M$, which cannot happen. This completes the proof.

2.E Bounded orbits: Let $\psi\left(x_{0}, \ldots, x_{n-1}\right)$ be a $(\max ,+)$-function and put $\bar{x}_{0}=\left(x_{0}, \ldots, x_{n-1}\right)$. We regard it as a map $\psi: \mathbb{R}^{n} \rightarrow \mathbb{R}^{n}$ by $\psi\left(\bar{x}_{0}\right)=\left(x_{1}, \ldots, x_{n-1}, \psi\left(\bar{x}_{0}\right)\right)$.

Let us say that $\psi$ is contracting, if there is some $0<\mu<1$ and some $l \geq 1$ so that for $\varphi=\psi^{l}$, the estimates:

$$
\left|\varphi\left(x_{0}, \ldots, x_{n-1}\right)\right| \leq(1-\mu)\left|\left(x_{0}, \ldots, x_{n-1}\right)\right|
$$

hold for all $\left(x_{0}, \ldots, x_{n-1}\right) \in \mathbb{R}^{n}$.

Let $f_{t}$ be the corresponding elementary function to $\psi$. Then the rational dynamics show boundedness for their orbits, whose property is invariant under tropical deformations: 
Proposition 2.3. Suppose $\psi$ is contracting. Then any orbits of $f_{t}$ are bounded away from both zero and infinity for all $t>1$.

Moreover there is some constant $C$ independent of $t$ and initial values, so that for any orbits $\left\{z_{N}\right\}_{N}$, there is some $N_{0}$ and for all $N \geq N_{0}$, the estimates hold:

$$
C^{-1} \leq z_{N} \leq C
$$

Proof: For any initial value $\left(z_{0}, \ldots, z_{n-1}\right)$, let us consider the orbit $\left\{z_{N}\right\}_{N}$ for $f_{t}$. We put the orbits $\left\{x_{N}\right\}_{N}$ and $\left\{x_{N}^{\prime}\right\}_{N}$ for $\psi$ and $\psi_{t}$ respectively, where $x_{0}=x_{0}^{\prime}=\log _{t} z_{0}, \ldots, x_{n-1}=x_{n-1}^{\prime}=\log _{t} z_{n-1}$. Then $x_{N}^{\prime}=\log _{t} z_{N}$ hold for all $N \geq 0$. Let us put $\varphi_{t}=\psi_{t}^{l}$ as $\varphi=\psi^{l}$.

Let us denote the components of $\varphi$ by $\left(\varphi^{0}, \ldots, \varphi^{n-1}\right) . \varphi^{i}$ has the form $x_{l+i}=\varphi^{i}\left(x_{0}, \ldots, x_{n}\right), 0 \leq i \leq n-1$, where $\varphi^{i}$ are also given by some compositions by $\psi$. Since compositions of $(\max ,+)$ functions are also $(\max ,+)$-type, it follows that $\varphi^{i}$ are also $(\max ,+)$ functions. Notice the equalities $\varphi_{t}^{i}\left(x_{0}, \ldots, x_{n-1}\right)=x_{l+i}^{\prime}$, where $\varphi_{t}=\left(\varphi_{t}^{0}, \ldots, \varphi_{t}^{n-1}\right)$.

Let $o \geq 1$ be the maximum number of the components among $\varphi^{i}$ and put $m=o^{n}$. Then by lemma 2.1(1), the estimates $\mid \varphi_{t}\left(x_{0}, \ldots, x_{n-1}\right)-$ $\varphi\left(x_{0}, \ldots, x_{n-1}\right) \mid \leq \log _{t} m$ hold.

Let us choose a small $\mu^{\prime}>0$ so that $\epsilon \equiv \mu-\mu^{\prime}>0$ is still positive. Let us put $\log _{t} m=\alpha \mu^{\prime}, t=m^{\frac{1}{\alpha \mu^{\prime}}}$ for some $\alpha>0$. Then by the above estimate,

$$
\left|\varphi_{t}\left(x_{0}, \ldots, x_{n-1}\right)\right| \leq(1-\mu)\left|\left(x_{0}, \ldots, x_{n-1}\right)\right|+\alpha \mu^{\prime}
$$

holds.

Let us put $\bar{p}_{N}=\varphi_{t}^{N}\left(x_{0}, \ldots, x_{n-1}\right) \in \mathbb{R}^{n}$. Suppose $\bar{p}_{N}$ satisfy $\left|\bar{p}_{N}\right|>\alpha$ for some $N \geq 0$. Then the above implies the estimate:

$$
\left|\varphi_{t}\left(\bar{p}_{N}\right)\right| \leq(1-\epsilon)\left|\bar{p}_{N}\right|
$$

We claim that there is some $N_{0} \geq 0$ and a constant $C$ independent of $t$ so that all points $\bar{p}_{N}$ satisfy uniform bounds $\left|\bar{p}_{N}\right| \leq C \alpha$ for all $N \geq N_{0}$. By the above contracting property, it is enough to show this when $\left|\bar{p}_{N}\right| \leq \alpha$ holds. Then let $o \geq 1$ be as above. $\varphi_{t}^{i}$ are of the forms 
$\log _{t}\left(t^{L_{1}^{i}}+\cdots+t^{L_{o^{\prime}}^{i}}\right)$, where $L_{j}^{i}$ are linear functions and $o^{\prime} \leq o$. Suppose $\max \left(L_{1}^{i}\left(\bar{p}_{N}\right), \ldots, L_{o^{\prime}}^{i}\left(\bar{p}_{N}\right)\right)=L_{1}^{i}\left(\bar{p}_{N}\right)$. Then the estimate holds:

$$
\begin{aligned}
\left|\varphi_{t}^{i}\left(\bar{p}_{N}\right)\right| & =\left|L_{1}^{i}\left(\bar{p}_{N}\right)+\log _{t}\left(1+t^{L_{2}^{i}\left(\bar{p}_{N}\right)-L_{1}^{i}\left(\bar{p}_{N}\right)}+\cdots+t^{L_{o^{\prime}}^{i}\left(\bar{p}_{N}\right)-L_{1}^{i}\left(\bar{p}_{N}\right)}\right)\right| \\
& \leq\left|L_{1}^{i}\left(\bar{p}_{N}\right)\right|+\log _{t} O
\end{aligned}
$$

Since $L_{j}^{i}$ are linear, it is enough to check $\log _{t} O \leq C \alpha$ for some $C$. Since $t=m^{\frac{1}{\alpha \mu^{\prime}}}$ hold, one can choose a large $C$ so that $o \leq m^{\frac{C}{\mu^{\prime}}}=t^{C \alpha}$. This verifies the claim.

Now $p_{N}^{i}=x_{N l+i}^{\prime}$ hold for $\bar{p}_{N}=\left(p_{N}^{0}, \ldots, p_{N}^{n-1}\right)$. Since the equality $z_{N}=t^{x_{N}^{\prime}}=m^{\frac{x_{N}^{\prime}}{\alpha \mu^{\prime}}}$ holds, the orbits:

$$
m^{\frac{-C}{\mu^{\prime}}} \leq z_{N l+i} \leq m^{\frac{C}{\mu^{\prime}}}, \quad N \geq N_{0}, \quad(0 \leq i \leq n-1)
$$

are bounded from both below and above, which are independent of $t$. If $l \leq n$, then we are done.

Suppose $l>n$. Then we regard $\left(z_{n}, \ldots, z_{2 n-1}\right)$ as the initial value, and apply the above estimates. Then $m^{\frac{-C}{\mu^{\prime}}} \leq z_{N l+i+n} \leq m^{\frac{C}{\mu^{\prime}}}$ hold for $0 \leq i \leq n-1$ and all $N \geq N_{1}>>0$. We iterate the same argument for the initial values $\left(z_{k n}, \ldots, z_{(k+1) n-1}\right), k=0,1, \ldots, s=\left[\frac{l}{n}\right]$, and obtain the same bounds for all $N \geq N_{k}>>0$.

Finally we put $L=\max \left(N_{0}, \ldots, N_{s}\right)$. Then the conclusion follows for all $N \geq L$. This completes the proof.

Remark: The above estimates show that on small neighbourhoods of 1 , some fluctuation of orbits $\left\{z_{N}\right\}_{N}$ occurs. They give us no information on the bahaviour of the orbits near 1 , and they will not converge to 1 in general.

For our later purpose, let us generalize proposition 2.3. We consider two cases. Let $\psi$ be a $(\max ,+)$ function. Let us say that $\psi$ is:

(1) eventually contracting, if there is some $0<\mu<1$ and $m_{0}, l$ so that for any initial value $\left(x_{0}, \ldots, x_{n-1}\right)$, there is some $m \leq m_{0}$ and the estimates hold for all $i=0,1,2, \ldots$ :

$$
\mid \psi^{l i}\left(\psi_{t}^{m}\left(\left(x_{0}, \ldots, x_{n-1}\right)\right)\left|\leq(1-\mu)^{i}\right| \psi_{t}^{m}\left(x_{0}, \ldots, x_{n-1}\right) \mid .\right.
$$


(2) essentially contracting, if there is some $0<\mu<1$ and $m_{0}, n_{0}$ so that for any initial value $\left(x_{0}, \ldots, x_{n-1}\right)$, there are $m \leq m_{0}$ and indices $l_{0}<l_{1}<l_{2}, \cdots \rightarrow \infty, l_{i+1}-l_{i} \leq n_{0}$, such that the estimates hold:

$$
\left|\psi^{l_{i}}\left(\psi_{t}^{m}\left(x_{0}, \ldots, x_{n-1}\right)\right)\right| \leq(1-\mu)^{i}\left|\psi_{t}^{m}\left(x_{0}, \ldots, x_{n-1}\right)\right|
$$

for all $i=0,1,2, \ldots$ We will say that it is essentially contracting with respect to $\left(\mu, m_{0}, n_{0}\right)$. Eventually contracting implies essential one.

Corollary 2.3. Suppose $\psi$ is essentially contracting. Then any orbits of $f_{t}$ are bounded away from both zero and infinity.

Moreover there is some constant $C$ independent of $t$ and initial values, so that for any orbits $\left\{z_{N}\right\}_{N}$, there is some $N_{0}$ and for all $N \geq N_{0}$, the estimates hold:

$$
C^{-1} \leq z_{N} \leq C
$$

Proof: For the eventually contracting case, one can choose the initial value $\psi_{t}^{m}\left(x_{0}, \ldots, x_{n-1}\right)$ rather than $\left(x_{0}, \ldots, x_{n-1}\right)$. Then the conclusion follows by proposition 2.3 .

Let us consider the essentially contracting case. Again one may replace the initial value by $\psi_{t}^{m}\left(x_{0}, \ldots, x_{n-1}\right)$. Let $\left\{x_{N}\right\}_{N},\left\{x_{N}^{\prime}\right\}_{N}$ and $\left\{z_{N}\right\}_{N}$ be orbits for $\psi, \psi_{t}$ and $f_{t}$ respectively. Then by the assumption, there are indices $l_{1}<l_{2}<\cdots \rightarrow \infty, l_{i+1}-l_{i} \leq n_{0}$, and $\left|\psi^{l_{i}}\left(x_{0}, \ldots, x_{n-1}\right)\right| \leq$ $(1-\mu)^{i}\left|\left(x_{0}, \ldots, x_{n-1}\right)\right|$ hold. Let us denote $\bar{x}_{N}^{\prime}=\left(x_{N}^{\prime}, \ldots, x_{N+n-1}^{\prime}\right)$.

We proceed the same argument as the proof of proposition 2.3. Let us denote $\psi^{l}=\left(\psi_{0}^{l}, \ldots, \psi_{n-1}^{l}\right)$ and denote $m_{l}^{i}$ as the number of the components of $\psi_{i}^{l}$. Then put $o=\max \left\{m_{l}^{i}: 0 \leq i \leq n-1,0 \leq l \leq\right.$ $\left.n_{0}+1\right\}$, and $m=o^{n}$. As before we put $\log _{t} m=\alpha \mu^{\prime}$. Then there is a constant $C$ and indices $N_{0}<N_{1}<\ldots, N_{i+1}-N_{i} \leq n_{0}+1$, so that $\left|\bar{x}_{N_{i}}\right| \leq C \alpha$ hold for all $i$. Since $N_{i+1}-N_{i}$ are uniformly bounded, it follows by replacing the constant by a larger $C^{\prime} \geq C$ if necessarily, that $\left|\bar{x}_{N}^{\prime}\right| \leq C^{\prime} \alpha$ hold for all $N \geq N_{0}$. Thus uniformity $C^{-1} \leq z_{N} \leq C$ hold for all $N \geq N_{0}$.

This completes the proof.

2.F Unbounded orbits: Let $\varphi$ be a $(\max ,+)$-function on $\mathbb{R}^{n}$. Let us say that it is homogeneous, if $\varphi\left(\alpha x_{0}, \ldots, \alpha x_{n-1}\right)=\alpha \varphi\left(x_{0}, \ldots, x_{n-1}\right)$ hold for all $\alpha \in \mathbb{R}$ and $\left(x_{0}, \ldots, x_{n-1}\right) \in \mathbb{R}^{n}$. 
Let us take initial values $\bar{x}_{0}=\left(x_{0}, \ldots, x_{n-1}\right) \in \mathbb{R}^{n}$ and denote the orbits by $x_{N}$ defined by $\varphi$. Then for a homogeneous $\varphi$, let us put:

$$
L(N)=\inf _{\left|\bar{x}_{0}\right|=1} \sup _{l \leq N}\left\{x_{l} ; x_{n+i}=\varphi\left(x_{i}, \ldots, x_{n+i-1}\right)\right\} .
$$

Let us say that the dynamics by $\varphi$ is positively unbounded, if there is some $N_{0}$ with $L\left(N_{0}\right)>1$ holds.

Let $f_{t}$ be the corresponding rational function and $z_{n+i}=f_{t}\left(z_{i}, \ldots, z_{n+i-1}\right)$ be the dynamics.

Lemma 2.4. Suppose $\varphi$ is positively unbounded. Then there is a constant $C$ so that for any $g_{t}$ of tropical deformation of $f_{t}, g_{t}$ have unbounded orbits for any initial values $\bar{z}_{0}$ with $\left|\bar{z}_{0}\right| \geq m^{C}$, where $m$ is the number of the components of $g_{t}$.

Proof: Let us denote the (max, + )-function corresponding to $g_{t}$ by $\psi$. $\psi$ is also positively unbounded. By the assumption, there is some $N_{0}$ with $L\left(N_{0}\right)>1+\delta$ holds for some $\delta>0$. Let us put the number of the components of $g_{t}$ by $m=m_{g_{t}}$. Let $\left\{x_{N}\right\}_{N}$ and $\left\{x_{N}^{\prime}\right\}_{N}$ be the orbits of $\psi$ and $\psi_{t}$ with the same initial value $\bar{x}_{0}=\left(x_{0}, \ldots, x_{n-1}\right)$ respectively. Then by lemma 2.2, there is some constant $C$ independent of choice of $g_{t}$ so that the corresponding orbit satisfies the estimates $\left|x_{i}-x_{i}^{\prime}\right|<$ $C \log _{t} m$ for $0 \leq i \leq N_{0}$.

Now choose any initial value $\bar{x}_{0}$ with $\frac{\delta}{2}\left|\bar{x}_{0}\right|>C \log _{t} m$. By the assumption, there is some $n_{0} \leq N_{0}$ so that $x_{n_{0}} \geq\left|\bar{x}_{0}\right|(1+\delta)$ holds. Then we have the estimates:

$$
x_{n_{0}}^{\prime}>\left|\bar{x}_{0}\right|(1+\delta)-\log _{t} m^{C}>\left|\bar{x}_{0}\right|\left(1+\frac{\delta}{2}\right) .
$$

Now we choose another initial value $\bar{x}_{0,1}=\left(x_{n_{0}}^{\prime}, \ldots, x_{n_{0}+n-1}^{\prime}\right)$, and denote the orbits as $x_{N, 1}^{\prime}$ and $x_{N, 1}$ for $\psi_{t}$ and $\psi$ respectively, $N=0,1, \ldots$ Then since $\psi$ is homogeneous, there is $0 \leq n_{1} \leq N_{0}$ so that $x_{n_{1}, 1} \geq$ $\left|\bar{x}_{0}\right|(1+\delta)\left(1+\frac{\delta}{2}\right)$ holds. Thus the estimate:

$$
x_{n_{1}, 1}^{\prime}>\left|\bar{x}_{0}\right|(1+\delta)\left(1+\frac{\delta}{2}\right)-\log _{t} m^{C}>\left|\bar{x}_{0}\right|(1+\delta)
$$

holds. Notice $x_{n_{1}, 1}^{\prime}=x_{n_{0}+n_{1}}^{\prime}$ lies on the orbits of $\psi_{t}$ with the initial value $\left(x_{0}, \ldots, x_{n-1}\right)$. 
Let us iterate the same process. Let $\bar{x}_{0,2}=\left(x_{n_{1}, 1}^{\prime}, \ldots, x_{n_{1}+n-1,1}^{\prime}\right)$ be another initial value, and denote the orbits as $x_{N, 2}^{\prime}$ and $x_{N, 2}$ for $\psi_{t}$ and $\psi$ respectively, $N=0,1, \ldots$ Then there is $0 \leq n_{2} \leq N_{0}$ so that $x_{n_{2}, 2} \geq$ $\left|\bar{x}_{0}\right|(1+\delta)(1+\delta)$, and so $x_{n_{2}, 2}^{\prime}>\left|\bar{x}_{0}\right|(1+\delta)^{2}-\log _{t} m^{C}>\left|\bar{x}_{0}\right|\left(1+\frac{3}{2} \delta\right)$ hold, where $x_{n_{2}, 2}^{\prime}=x_{n_{0}+n_{1}+n_{2}}^{\prime}$. The same process gives $x_{n_{k}, k}^{\prime}$ with the estimates $x_{n_{k}, k}^{\prime}>\left|\bar{x}_{0}\right|\left(1+\frac{(k+1) \delta}{2}\right), k=0,1, \ldots$, and $x_{n_{k}, k}^{\prime}=x_{\sum_{i=0}^{k} n_{i}}^{\prime}$.

Now all points $x_{n_{k}, k}^{\prime}$ lie on the orbits $\left\{x_{n}^{\prime}\right\}_{n}$ by $\psi_{t}$ with the initial value $\left(x_{0}, \ldots, x_{n-1}\right)$. Let us put $z_{N}=t^{x_{N}^{\prime}}$. Then the bound $\left|\bar{x}_{0}\right|>\log _{t} m^{C}$ follows if $z_{i}>m^{C}$ hold for some $0 \leq i \leq n-1$. Since $\left\{z_{N}\right\}_{N}$ is the orbit for $g_{t}$, the result follows. This completes the proof.

Remark: When $\varphi$ is homogeneous, the corresponding $f_{t}$ is not necessarily $t$ independent. For example consider $\varphi(x)=\max (2 x,-2 x,-1+x)$.

\section{Rational perturbation of dynamics in two vari- ables}

3.A Traces: Let $\varphi$ be a $(\max ,+)$-function by two variables. Let $\bar{x}_{0}=$ $\left(x_{0}, x_{1}\right) \in \mathbb{R}^{2}$ be initial values, and denote the orbits of $\varphi$ by $\left\{x_{N}\right\}_{N}$. Let us plot the sequence of points $\left(x_{N}, x_{N-1}\right) \in \mathbb{R}^{2}$, and regard $\varphi$ as a map:

$$
\varphi:\left(x_{N-1}, x_{N}\right) \rightarrow\left(x_{N}, x_{N+1}\right) \quad N \geq 1 .
$$

Let us denote $\varphi^{i}\left(x_{0}, x_{1}\right) \equiv \bar{x}_{i}$ for $i=0,1, \ldots$ Let $L_{0}$ be the straight line which connects $\bar{x}_{0}$ and $\bar{x}_{1}$ in $\mathbb{R}^{2}$. The trace of $\varphi$ with the initial value $\bar{x}_{0}$ is a connected piecewise linear line $L$ in $\mathbb{R}^{2}$ :

$$
L=\cup_{i \geq 0} \varphi^{i}\left(L_{0}\right) \subset \mathbb{R}^{2} .
$$

$L$ contains all the points $\cup_{i \geq 0} \bar{x}_{i} \subset L$.

For later purpose let us explicitly construct traces. Let $\varphi$ be of the form $\varphi(x, y)=\max \left(\alpha_{1}(x, y), \ldots, \alpha_{m}(x, y)\right)$, where $\alpha_{j}$ are linear functions.

Suppose $\varphi\left(\bar{x}_{0}\right)=\alpha_{j}\left(\bar{x}_{0}\right)$ for some $1 \leq j \leq m$. If the equality $\varphi(\bar{x})=$ $\alpha_{j}(\bar{x})$ hold for any point $\bar{x} \in L_{0}$, then $\varphi\left(L_{0}\right)$ is a straight line. 
Otherwise, let $l_{0}^{1} \subset L_{0}$ be a subline with one end point $\bar{x}_{0}$ so that the equality $\varphi(\bar{x})=\alpha_{j}(\bar{x})$ hold for any point $\bar{x} \in l_{0}^{1}$. We choose $l_{0}^{1}$ of maximal length with this property, and denote the other end point by $\bar{x}_{0}^{1}$. We put $j=j_{0}$.

Then there is another $j_{1} \neq j_{0}$ so that $\varphi\left(\bar{x}_{0}^{1}\right)=\alpha_{j_{1}}\left(\bar{x}_{0}^{1}\right)$ holds. Let $\bar{x}_{0}^{2}$ be another point on $L_{0}$ so that the equality $\varphi(\bar{x})=\alpha_{j_{1}}(\bar{x})$ hold for any points $\bar{x} \in l_{0}^{2}$, where $l_{0}^{2}$ is a subline on $L_{0} \backslash l_{0}^{1}$ which connects $\bar{x}_{0}^{1}$ and $\bar{x}_{0}^{2}$. Again we choose $l_{0}^{2}$ of maximal length with this property. Then one finds another $j_{2} \neq j_{1}$ so that $\varphi\left(\bar{x}_{0}^{2}\right)=\alpha_{j_{2}}\left(\bar{x}_{0}^{2}\right)$ holds, and we seek for $\bar{x}_{0}^{3}$ by the same way. By iterating this process, finally one divides $L_{0}$ into smaller sublines $L_{0}=l_{0}^{1} \cup l_{0}^{2} \cup \cdots \cup l_{0}^{k}$ for some $k$, where one end point of $l_{0}^{k}$ is $\bar{x}_{1}$. By the construction, the images $l_{1}^{j} \equiv \varphi\left(l_{0}^{j}\right)$ are all straight lines. Then we have a broken line as a union of line segments:

$$
L_{0} \cup \varphi\left(L_{0}\right)=\cup_{i=0}^{k} l_{0}^{i} \cup_{i=0}^{k} l_{1}^{i} .
$$

We do the same process, by replacing the role of $L_{0}$ by $l_{1}^{i}$, of dividing all $l_{1}^{i}$ into smaller sublines $l_{1}^{i}=\cup_{j=0}^{k^{\prime}} l_{1}^{i, j}$. Then again the images $l_{2}^{i, j} \equiv \varphi\left(l_{1}^{i, j}\right)$ are all straight lines, and we have a broken line as a union of line segments:

$$
L_{0} \cup \varphi\left(L_{0}\right) \cup \varphi^{2}\left(L_{0}\right)=\cup_{i} l_{0}^{i} \cup_{i, j} l_{1}^{i, j} \cup l_{2}^{i, j} .
$$

By this way one obtains $L$ by continuing this process possibly infinitely many times.

3.B Return maps for traces: Let $L$ be the trace for $\varphi$ with an initial point $(0,0) \neq \bar{x} \in \mathbb{R}^{2}$, and $M$ be the half infinite straight line containing the origin and $\bar{x}$. The return map for $L$ is an assignment:

$$
r(\bar{x}) \in M \cap L
$$

where on the connected subline $C \subset L$ along the iteration of $\varphi$, between $\bar{x}$ and $r(\bar{x}), C \cap M$ consists of only these two end points. $r^{2}(\bar{x}) \in C^{\prime} \cap L$ is another assignment, where on the connected subline $C^{\prime} \subset L$ along the iteration of $\varphi$, between $r(\bar{x})$ and $r^{2}(\bar{x}), C^{\prime} \cap M$ consists of only these two end points. $r^{k}(\bar{x})$ are similar.

In this paper we usually choose $M$ as the $x$-axis $[0, \infty) \times\{0\}$. 
Throughout the rest of section 3, we always assume that $\varphi$ are homogeneous.

Let $L$ be the trace of $\varphi$ with the end point $(1,0)$, and suppose $r^{k}(1,0) \neq$ $\phi$. Then there is some $n_{0} \geq 1$ so that $r^{k}(1,0) \in \varphi^{n_{0}}\left(L_{0}\right)$. For two points $\bar{x}, \bar{y} \in L$, let us denote by $l(\bar{x}, \bar{y}) \subset L$ the broken segment along the iteration of $\varphi$, connecting these two points.

Let $S_{k}$ be the straight line connecting $(1,0)$ and $r^{k}(1,0)$ on the $x$-axis, and denote the circle $s_{k}=l\left((1,0), r^{k}(1,0)\right) \cup S_{k} \subset \mathbb{R}^{2}$. Let us say that $s_{k}$ is non trivial, if it is not contractible in $\mathbb{R}^{2} \backslash\{(0,0)\}$.

Now $\varphi^{n_{0}}\left(L_{0}\right)$ splits as two broken segments:

$$
l\left(\varphi^{n_{0}}(1,0), r^{k}(1,0)\right) \cup l\left(r^{k}(1,0), \varphi^{n_{0}+1}(1,0)\right)
$$

and denote the connected broken line:

$$
C_{k}=l\left(r^{k}(1,0), \varphi^{n_{0}+1}(1,0)\right) \cup \varphi\left(l\left(\varphi^{n_{0}}(1,0), r^{k}(1,0)\right)\right) \subset L .
$$

Lemma 3.1. Suppose $C_{k} \neq \phi$ for some $k$. Then for any $\bar{x} \in L_{0}$, there is some $\bar{x}^{\prime} \in C_{k}$ and $a>0$ so that $\bar{x}=a \bar{x}^{\prime}$.

Proof: The end points of $C_{k}$ is $r^{k}(1,0)=(b, 0)$ and $\varphi\left(r^{k}(1,0)\right)=\varphi(b, 0)$ for some $b>0$. Thus it is a broken line connecting $(b, 0)$ and $\varphi(b, 0)$. Since $L_{0}$ is a segment connecting $(1,0)$ and $\varphi(1,0)$, and since $\varphi$ is homogeneous, thus the conclusion clearly follows. This completes the proof.

We say that $\varphi$ is focus, if there is some $k \geq 1$ so that $\bar{x} \in a L_{0}$ hold for some $a>0$, for any points $\bar{x} \in C_{k} \neq \phi$.

Any homogeneous and recursive maps are focus.

Let us define the distances:

$$
\begin{aligned}
& D_{i}^{k}(\varphi)=\inf \left\{|\bar{y}|-|\bar{x}|: \bar{x} \in C_{k}, \bar{y} \in L_{0}\right\}, \\
& D_{s}^{k}(\varphi)=\sup \left\{|\bar{y}|-|\bar{x}|: \bar{x} \in C_{k}, \bar{y} \in L_{0}\right\} .
\end{aligned}
$$

We call the degree of $\varphi$ as the minimum $k \geq 1$ so that (1) $\varphi$ is focus with respect to $k,(2) s_{k}$ is non trivial, and (3) one of $D_{i}^{k} \geq 0$ or $D_{s}^{k} \leq 0$ hold. 
If there are no such $k$, then degree of $\varphi$ is 0 .

$\varphi(x, y)=\max (0, y)-x$ is recursive of period 5 , and its degree is 1 . $\varphi(x, y)=\max (-y, y)-x$ is recursive of period 9 , and its degree is 2 .

Definition 3.1. Let $\varphi$ be focus of degree $k \geq 1$.

(1) It is stably focus, if the estimate $D_{i}^{k}(\varphi)>0$ is satisfied.

(2) It is unstable focus, if $D_{s}^{k}(\varphi)<0$ is satisfied.

Let $\varphi$ be a homogeneous (max, + )-function and $f_{t}$ be the corresponding elementary function. Now we consider tropical correspondence of dynamics:

Proposition 3.1. Suppose $\varphi$ is stably focus. Then any orbits of $f_{t}$ are bounded away from both zero and infinity.

Moreover there is some constant $C$ independent of $t$ and initial values, so that for any orbits $\left\{z_{N}\right\}_{N}$, there is some $N_{0}$ and for all $N \geq N_{0}$, the estimates hold:

$$
C^{-1} \leq z_{N} \leq C
$$

Proof: Let $k \geq 1$ be the degree of $\varphi$, and $C_{k}$ and $n_{0}$ be as above.

Let $\left(z_{0}, z_{1}\right)$ be any initial value, and put $x_{0}=\log _{t} z_{0}, x_{1}=\log _{t} z_{1}$. Then we have orbits $\left\{x_{N}\right\}_{N},\left\{x_{N}^{\prime}\right\}_{N},\left\{z_{N}\right\}_{N}$ for $\varphi, \varphi_{t}, f_{t}$ respectively.

By lemma 2.2, there is some constant $C$ invariant under tropical deformations so that $\left|x_{i}-x_{i}^{\prime}\right| \leq \log _{t} m^{C}$ for all $0 \leq i \leq n_{0}+1$, where $m$ is the number of the components of $f_{t}$.

It follows from homogeneity of $\varphi$ and the above estimates that if initial values $\bar{x}_{0}$ satisfy the estimates $\left|\bar{x}_{0}\right| \geq c \log _{t} m^{C}$ for large $c>>1$, then there is some $a>0,0 \leq m \leq n_{0}+1$ so that $\bar{y}_{0}=\varphi_{t}^{m}\left(\bar{x}_{0}\right)$ lies in $\log _{t} m^{C}$ neighbourhood of $a L_{0}$, where $a L_{0}$ is a scale change of $L_{0}$ by $a$.

Now we claim that if $\left|\bar{x}_{0}\right| \geq c \log _{t} m^{C}$ holds, then there is a constant $1>\mu>0$ and some $n_{0}$ so that $\varphi$ is essentially contracting with respect to $\left(\mu, n_{0}+1, n_{0}+2\right)$.

On the other hand if $\max \left(z_{i}, z_{i}^{-1}\right) \geq m^{C c}$ hold for one of $i=0,1$, then the estimate $\left|\bar{x}_{0}\right| \geq c \log _{t} m^{C}$ follows. Combining with these, the conclusion follows by corollary 2.3 . 
Let us verify the claim. Assume $\left|\bar{x}_{0}\right| \geq c \log _{t} m^{C}$ holds for large $c$ and choose $m$ and $\bar{y}_{0}$ as above. Then for $n_{0}-1 \leq p \leq n_{0}+2, \bar{y}=\varphi^{p}\left(\bar{y}_{0}\right) \in$ $a C_{k}$. Since we have chosen a large $c$, it follows from homogeneity that there is some $0<\mu<1$ so that $|\bar{y}| \leq(1-\mu)\left|\bar{y}_{0}\right|$ holds.

Next by the assumption, $\bar{y} \in c L_{0}$ for some $c>0$. Then it follows again by homogeneity that for $p^{\prime}=n_{0}$ or $n_{0}+1, \varphi^{p^{\prime}}(\bar{y})=\varphi^{p+p^{\prime}}\left(\bar{y}_{0}\right) \in c C_{k}$, and the estimate $\left|\varphi^{p+p^{\prime}}\left(\bar{y}_{0}\right)\right| \leq(1-\mu)\left|\varphi^{p}\left(\bar{y}_{0}\right)\right| \leq(1-\mu)^{2}\left|\bar{y}_{0}\right|$ holds.

One can iterate the second step to see that $\varphi$ is essentially contracting. This verifies the claim.

This completes the proof.

Remark: When $\varphi$ is recursive and not contracting, then the above proof gives no information on boundedness of orbits for the corresponding rational function.

3.C Perturbation of recursive maps: Let $\varphi$ be a $(\max ,+)$-function. A conserved polygon is a polygon $P \subset \mathbb{R}^{2}$ on which any orbits $\left(x_{n-1}, x_{n}\right)$ with $\left(x_{0}, x_{1}\right) \in P$ lie.

Conserved polygons satisfy that (1) when $\varphi$ is recursive, then closed traces are conserved polygons, and (2) if $\varphi$ is homogeneous, then they are scale invariant, in the sense that if $P$ is a conserved polygon, then $r P$ are also the same for any $r>0$.

Let $\varphi(x, y)$ be a $(\max ,+)$-function with two variables, and $\left|\epsilon_{i}\right|<<1$ be two small numbers, $i=0,1$. $\epsilon_{i}$ perturbation of $\varphi$ is given by:

$$
\varphi^{i}\left(x_{0}, x_{1}\right)=\varphi\left(x_{0}, x_{1}\right)+\epsilon_{i} x_{i} .
$$

Let $f_{t}\left(z_{0}, z_{1}\right)$ be a rational function. Correspondingly $\epsilon_{i}$ perturbation of $f_{t}$ is given by:

$$
f_{t}^{\epsilon_{i}}\left(z_{0}, z_{1}\right)=z_{i}^{\epsilon_{i}} f_{t}\left(z_{0}, z_{1}\right) .
$$

Let $\left(x_{0}, x_{1}\right) \in \mathbb{R}^{2}$ be an initial value, and $\left\{x_{n}\right\}_{n}$ be the orbits for $\varphi^{i}$. Throughout this section, we regard $\varphi^{i}$ as maps $\varphi^{i}:\left(x_{n-1}, x_{n}\right) \rightarrow$ $\left(x_{n}, x_{n+1}\right)$, and study distributions of the sequences of points $\left(x_{n-1}, x_{n}\right) \in$ $\mathbb{R}^{2}$. 
3.D A recursive map: Let us calculate an example. Let $z_{N+1}=$ $f\left(z_{N-1}, z_{N}\right)=\frac{1+z_{N}}{z_{N-1}}$ be the recursive map of period 5 . Its tropical transform is given by:

$$
x_{N+1}=\varphi\left(x_{N-1}, x_{N}\right)=\max \left(0, x_{N}\right)-x_{N-1}
$$

whose orbits also have all period 5 . Here we study dynamics given by $\epsilon_{0}$ perturbations of the homogeneous (max, + )-function:

$$
\varphi^{\epsilon}\left(x_{0}, x_{1}\right)=\max \left(0, x_{1}\right)-x_{0}+\epsilon x_{0} .
$$

Let $\left(x_{0}, x_{1}\right)=(1,0)$ be the initial value. Then the orbit of $\varphi$ is given by:

$$
(1,0),(0,-1),(-1,0),(0,1),(1,1)
$$

and the pentagon $P_{5}$ with the vertices above is mapped by $\varphi$ into itself. Thus $P$ is a conserved pentagon, and since the equation is homogeneous, any $r P$ are also the case for any $r \geq 0$.

Here we show the following:

Theorem 3.1. Let $g_{t}$ be any tropical deformation of $f(z, w)=\frac{1+w}{z}$. Then $\epsilon_{0}$ perturbations $g_{t}^{\epsilon}(z, w)=z^{\epsilon} g_{t}(z, w)$ are stationary.

For example, $g^{\epsilon}(z, w)=z^{\epsilon}\left(\frac{1+w}{z}+l \frac{1}{z}\right), l=0,1, \ldots$ are all the cases.

Thus we have the following properties:

(1) If $\epsilon>0$ is positive, then any iterations $\left\{z_{N}\right\}_{N}$ of $g_{t}^{\epsilon}$ are bounded away from both zero and infinity.

(2) If $\epsilon<0$ is negative, then any orbits of $g_{t}^{\epsilon}$ with large norms of initial values are unbounded.

Proof of theorem 3.1: Combinations of lemma 2.4 and proposition 3.1 with the following lemma verifies theorem 3.1. Let $|\epsilon|<<1$ be sufficiently small. Let $L_{\epsilon}$ be the traces of $\varphi^{\epsilon}$ with the end point $(1,0)$, and recall $C_{1} \subset L_{\epsilon}$ in 3.B. Then we have:

Lemma 3.2. For any small $\epsilon, \varphi^{\epsilon}$ are focus, and the degrees of $\varphi^{\epsilon}$ are equal to one. 
(1) If $\epsilon>0$, then $\varphi^{\epsilon}$ is stably focus, $r(1,0)<1$, and $C_{1}$ all lie in the interior of $P_{5}$ except $(1,0)$.

(2) if $\epsilon<0$, then it is unstably focus, $r(1,0)>1$, and $C_{1}$ all lie in the exterior of $P_{5}$ except $(1,0)$.

Proof: For any signs of $\epsilon, \varphi^{\epsilon}(1,0)=(0,-1+\epsilon)$ hold. Let $l_{\epsilon}$ be the segment connecting the points $(1,0)$ and $(0,-1+\epsilon)$, and consider the trace $L_{\epsilon}$ with the initial point $(1,0)$.

The direct calculation gives its orbit as:

$$
\begin{gathered}
p_{0}=(1,0), p_{1}=(0,-1+\epsilon), p_{2}=(-1+\epsilon, 0), p_{3}=\left(0,(1-\epsilon)^{2}\right), \\
p_{4}=\left((1-\epsilon)^{2},(1-\epsilon)^{2}\right), p_{5}=\left((1-\epsilon)^{2}, \epsilon(1-\epsilon)^{2}\right)
\end{gathered}
$$

for both cases of $\pm \epsilon>0$.

$p_{6}$ depends on signs of $\epsilon$. we have the following:

$$
p_{6}= \begin{cases}\left(\epsilon(1-\epsilon)^{2},(-1+2 \epsilon)(1-\epsilon)^{2}\right) & \epsilon>0 \\ \left(\epsilon(1-\epsilon)^{2},-(1-\epsilon)^{3}\right) & \epsilon<0\end{cases}
$$

Let $S_{\epsilon}$ be the broken lines which differ with respect to signs of $\epsilon$ as follows.

For $\epsilon \geq 0, r^{1}(1,0)$ is the intersection between $l\left(p_{5}, p_{6}\right)$ and the $x$-axis, and so $r^{1}(1,0)=\left((1-\epsilon)^{3}, 0\right)$. $S_{\epsilon}$ is a broken line connecting the eight points $\left\{p_{0}, \ldots, p_{6}, \varphi^{\epsilon}\left(r^{1}(1,0)\right)=\left(0,-(1-\epsilon)^{4}\right)\right\}$.

For $\epsilon<0, r^{1}(1,0)$ is the intersection between $l\left(p_{4}, p_{5}\right)$ and the $x$-axis, and so $r^{1}(1,0)=\left((1-\epsilon)^{2}, 0\right)$. $S_{\epsilon}$ is a broken line connecting the seven points $\left\{p_{0}, \ldots, p_{5}, \varphi^{\epsilon}\left(r^{1}(1,0)\right)=\left(0,-(1-\epsilon)^{3}\right)\right\}$.

In both cases, we claim the inclusions:

$$
C_{1} \subset S_{\epsilon} \subset L_{\epsilon} .
$$

Then it is immediate by drawing $S_{\epsilon}$ on the plane to see that it is inside $P_{5}$ for $\epsilon>0$ and outside for $\epsilon<0$, and the conclusions follow.

Now let us verify the claim. Notice that the bending points are $\varphi^{\epsilon}(x, y)$ with $y=0$. Firstly for any points on $L_{0}$, all $y$ components on the 
line are non positive. Thus $\varphi^{\epsilon}\left(L_{0}\right)$ is a straight line, $L_{0}=l_{0}^{0}$ and so $L_{0} \cup \varphi^{\epsilon}\left(L_{0}\right)=l_{0}^{0} \cup l_{1}^{0}$ in 3.A. For any points on $l_{1}^{0}=\varphi^{\epsilon}\left(L_{0}\right)$ also, all $y$ components on the line are non positive. Thus $\left(\varphi^{\epsilon}\right)^{2}\left(L_{0}\right)=l_{2}^{0,0}$ is also a straight line, where $l_{1}^{0}=l_{1}^{0,0}$. Next for any points on $l_{2}^{0,0}=\left(\varphi^{\epsilon}\right)^{2}\left(L_{0}\right)$, all $y$ components on the line are non negative. Thus $\left(\varphi^{\epsilon}\right)^{3}\left(L_{0}\right)$ is also a straight line.

By the same reasoning, $\left(\varphi^{\epsilon}\right)^{i}\left(L_{0}\right)$ are all straight lines for $0 \leq i \leq 5$ $(\epsilon \geq 0)$ and for $0 \leq i \leq 4(\epsilon<0)$.

Now for $\left.\epsilon \geq 0, l\left(p_{6}, \varphi^{\epsilon}\left(r^{1}(1,0)\right)\right)=\varphi^{\epsilon}\left(p_{5}, r^{1}(1,0)\right)\right)$ are straight lines, and for $\left.\epsilon<0, l\left(p_{5}, \varphi^{\epsilon}\left(r^{1}(1,0)\right)\right)=\varphi^{\epsilon}\left(p_{4}, r^{1}(1,0)\right)\right)$ are also the same.

These imply that $S_{\epsilon}$ are given by unions of segments, $\left(\varphi^{\epsilon}\right)^{i}\left(L_{0}\right)(0 \leq$ $i \leq 5)$ with $l\left(p_{6}, \varphi^{\epsilon}\left(r^{1}(1,0)\right)\right)$ for $\epsilon \geq 0$, and $\left(\varphi^{\epsilon}\right)^{i}\left(L_{0}\right)(0 \leq i \leq 4)$ with $l\left(p_{5}, \varphi^{\epsilon}\left(r^{1}(1,0)\right)\right)$ for $\epsilon<0$. Thus the inclusion $S_{\epsilon} \subset L_{\epsilon}$ hold for both cases, and so $C_{1} \subset S_{\epsilon}$ also hold.

This completes the proof.

\section{References}

[GKP] R.Graham, D.Knuth and O.Patashnik, Concrete mathematics, Addison-Wesley (1994).

[HY] R.HiRota And H.Yahagi, Recurrence equations, an integrable system, Journal of Phys. Soc. Japan 71 pp. 2867-2872 (2002).

[K1] T.KATO, Operator dynamics in molecular biology, in the Proceedings of the first international conference on natural computation, L.N. in computer science $\mathbf{3 6 1 1}$ pp. 974-989 (2005), Springer.

[K2] T.KATO, Pattern formation from projectively dynamical systems and iterations by families of maps, MPI preprint (2006).

[LM] G.Litvinov And V.Maslov, The correspondence principle for idempotent calculus and some computer applications, Idempotency, Ed. J.Gunawardena, Cambridge Univ. Press, pp420-443 (1998). 
[MS] W. De Melo And S. van Strien, One dimensional dynamics, Springer (1993).

[Mi] G.Mikhalkin, Amoebas and tropical geoemtry, in Different faces of geometry eds, S.Donaldson, Y.Eliashberg and M.Gromov, Kluwer academic plenum publ., (2004).

[R] C.Robinson, Dynamical systems, stability, symbolic dynamics, and chaos, Studies in Adv. Math., CRC press (1999).

[TI] D.TAKahashi AND M.IwaO, Geometrical dynamics of an integrable piecewise-linear mapping, Bilinear integrable systems: from classical to quantum, continuous to discrete, (NATO science series II: mathematics, physics and chemistry, eds. L.D.Faddeev, P.V. Moerbeke, F.Lambert) 201 pp. 291-300 (2005)

[V] O.VIro, Dequantization of real algebraic geometry on logarithmic paper, Proc. of the European Congrress of Math., (2000).

[W] S.Wolfram, Cellular automata and complexity, Addison Wesley (1994).

Tsuyoshi Kato

Department of Mathematics

Faculty of Science

Kyoto University

Kyoto 606-8502

Japan 